\title{
Correction to: New scheme for measurement-device-independent quantum key distribution
}

\author{
Lian Wang ${ }^{1} \cdot$ Yuan-Yuan Zhou ${ }^{1} \cdot$ Xue-Jun Zhou $^{1} \cdot$ Xiao Chen $^{1} \cdot$ Zheng Zhang $^{1}$ \\ Published online: 22 November 2018 \\ (c) Springer Science+Business Media, LLC, part of Springer Nature 2018
}

\section{Correction to: Quantum Information Processing (2018) 17: 231 https://doi.org/10.1007/s11128-018-1991-x}

A measurement-device-independent quantum key distribution (MDI-QKD) scheme with passive heralded single-photon sources has previously been published [1], while the two-intensity MDI-QKD scheme with a two-mode source proposed in our paper can been regarded as the extension and further research of the work [1].

This work should have been cited in our paper, and we would like to thank Qin Wang for bringing this important work to our attention.

\section{Reference}

1. Wang, Q., Zhou, X.Y., Guo, G.C.: Realizing the measure-device-independent quantum-key-distribution with passive heralded-single photon sources. Sci. Rep. 6, 35394 (2016)

The original article can be found online at https://doi.org/10.1007/s11128-018-1991-x.

Yuan-Yuan Zhou

Liuzh531@163.com

1 Department of Electronic Engineering, Naval University of Engineering, Wuhan 430033, China 